\title{
Erratum: Different ICT Competency but Similar Pattern between Students with/without Learning Disabilities?
}

\author{
Ming-Chung Chen ${ }^{1}$, Chen-Ming Chen ${ }^{1}$, Ya-Ping $\mathrm{Wu}^{1}$, \\ Chien-Chuan $\mathrm{Ko}^{2}$, and Yao-Ming $\mathrm{Yeh}^{3}$ \\ ${ }^{1}$ Department of Special Education, National Chiayi University, Chiayi, Taiwan \\ tomson2@ms18. hinet. net, \\ \{mtchen, ping78kimo\} @mail.ncyu.edu. tw \\ ${ }^{2}$ Department of Computer Science and Information Engineering, \\ National Chiayi University, Chiayi, Taiwan \\ kocc@mail.ncyu.edu.tw \\ ${ }^{3}$ Department of Computer Science and Information Engineering, \\ National Taiwan Normal University, Taiwan \\ ymyehaice.ntnu.edu.tw
}

K. Miesenberger et al. (Eds.): ICCHP 2014, Part II, LNCS 8548, pp. 528-531, 2014.

(C) Springer International Publishing Switzerland 2014

\section{DOI 10.1007/978-3-319-08599-9_89}

Of the paper "Different ICT Competency but Similar Pattern between Students with/without Learning Disabilities?" on pages 528-531 of this publication, the name of the author Ting-Fang $\mathrm{Wu}$ was inadvertently omitted in the originally published version. The order of the authors and affiliations should read as below:

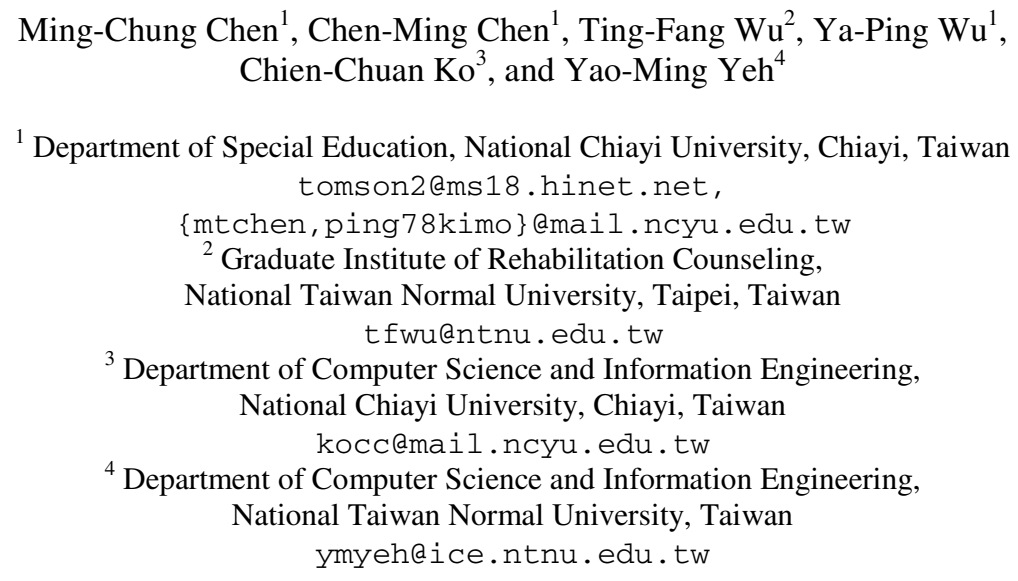

The original online version for this chapter can be found at http://dx.doi.org/10.1007/978-3-319-08599-9_78 